\title{
Research on Mobile Applications Interaction Design Based on Cognitive Ability of the Elderly
}

\author{
Lin Lin \\ Art Design \& Creative Industry, Nangfang College of Sun Yat-sen University \\ Guangzhou, China \\ 297254729@qq.com
}

\begin{abstract}
With the popularization of intelligent devices, more and more old people will become the consumers of mobile application. This paper firstly discusses the cognitive characteristics of the elderly. The cognitive ability of the elderly is characterized by a decrease in comprehension and performance. By analyzing the status of using mobile applications by the elderly, it is found that the mobile applications have problems that the function module is complex, the interaction logic is not consistent with the behavior logic of the elderly and the interface design is not suitable for the elderly. By studying the interaction design method and the change of cognitive ability of the elderly, this paper presents an interactive design method for elderly mobile applications.
\end{abstract}

Keywords-Elderly; Cognitive ability; Mobile applications; Interaction design

\section{INTRODUCTION}

With the popularization of smart mobile terminal and the rapid development of mobile internet, the use of mobile phones has become more and more frequent. As a tool, mobile phone can meet people's requirements and needs, and improve people's quality of life. As the elderly population increases, the frequency of mobile phone use of elderly increases either, and most older people recognize the role of mobile phones as tools for communication, entertainment and social interaction, and feel positive about the use of them for safely [1].

The mobile application, taking the mobile phone as carrier, is the main means for mobile phones to improve their functions and provide users with richer experience. With the improvement of people's quality of life and the increase of living demand, more and more types of mobile phone applications have become a flood. However, the main target users of existing apps are young people, there are few applications specifically developed for the elderly, and those for the elderly are not applicable to them. The main reason is that without considering the physiological and psychological characteristics of old people, interaction process and interaction of mobile phones for the elderly user should be different from those designed for the young. Therefore, starting from the cognitive ability in the elderly, this article studies the old phone interaction design method, which can promote the development of the elderly interaction design, also can help the elderly to enjoy using mobile devices.

\section{ANALYSIS OF COGNITIVE ABILITIES OF THE ELDERLY}

Cognitive abilities include intelligence, attention and alertness, speech, memory and learning function, visual spatial ability, mental motor ability and executive function. In modern cognitive psychology, the human cognition is studied from the viewpoint of information processing [2], the cognitive characteristics of the elderly are reflected in information perception ability, information processing ability, information anti-interference ability and information sensitivity [3]. The general trend of cognitive ability of the elderly is decline and aging, which is mainly reflected in both comprehension and executive ability in the daily life.

\section{A. Comprehension Ability of the Elderly}

Comprehension Ability is a measurement of the understanding of things. The change of comprehension ability of old people is represented by intelligence and memory.

Intelligence can be divided into language skills and the fast learning ability. The language ability is acquired through acquired knowledge, which is associated with the accumulation of cultural knowledge and understanding ability, and can be constantly trained to preserve the cognitive function. And the fast learning ability is greatly decreases with age, at this time, the brain slows down information processing. And the effect of the decline, for the elderly, is that they will show great difficulty in quickly making a response or completing the task. Therefore, for the elderly, the ability to analyze and judge things maintain at normal level, but the reaction rate of new things will decrease.

Memory appears in apparent recession after 60, the elderly will easier to forget what happened recently, and have longterm memory of things happened before the recession. The elderly will be to have no confidence in learning new things, and even produce psychological resistance. Also, the phenomenon of memory impairment, mental solidification and concentration difficulty is the manifestation of the decline of comprehension ability of the elderly [4]. 


\section{B. Executive Function of the Elderly}

The Executive Function of the elderly is determined by the ability of visual, auditory, tactile and other physical sensations. In visual perceptions, the elderly used to suffer symptoms of reduced sensitivity to color, drops to perception of the graphics and poor judgment of object space relations, etc., and some elderly have eye diseases like cataracts and glaucoma. In auditory aspect, the elderly are less sensitive to sound, they need a stronger voice intensity than normal young people need to achieve the equal level. The decline of auditory function not only influences the elderly to access to information, at the same time also affects the voice expression of the elderly. In tactile aspect, along with the aging body function, bones, muscles, joints and the skin of the elderly will appear different degrees of aging, which caused muscle weakness, decreased flexibility and reduced tactility, therefore the elderly are prone to errors in practical operation in daily life.

\section{Status OF THE ElDERly Using MobILE APPLICATION}

\section{A. Functional needs of the elderly using mobile application}

At present, mobile smart terminals on the market mainly use mobile phones as carriers, and use applications to realize various functions. In terms of functional requirements, the applications used by the elderly users can be classified into two categories. One is the app that solves the basic problems in life. These applications can be classified into the categories of:

- Utilities: mobile phone offers many functions that make life convenient, in day-to-day life the elderly have a lot of tooling needs, such as magnifying glass, calculators and dictionaries, etc. Using mobile phone is far more convenient than in reality to find the corresponding tools. Thus, even to some elderly users who don't often use a mobile phone, tool applications like a flashlight, a magnifying glass, calculator, input method, email, dictionary and translation, etc., are very practical.

- Lifestyle class: with the realization of intelligent life, the elderly are becoming more and more accepting of intelligent life, and there are also demands for information. Therefore, lifestyle apps such as weather, medical, transportation, banking, cookbook and reminder can provide substantial help for the elderly.

The other app that caters to the interests of the elderly and meets the social needs of them can be classified into the categories of:

- Social: more and more people use mobile devices to communicate. Social applications such as WeChat and Tencent QQ is the main social applications, target users of this kind of apps cover all kinds of people. In order to satisfy the psychological needs of communication with children, relatives and neighbors, more and more old people are joining the group of using social applications.

- News: As marginalized people, the elderly hope to be exposed to more news and topics in the hope of communication. Therefore, the information apps can meet their needs very well, such as news apps, BBS apps, audio and video apps, broadcast apps, all of which are popular with the elderly.

- Entertainment: Old people have plenty of time to play games. Proper games not only allow the elderly to enjoy the fun of the game, but also make the old people connect with the times. Compared with the diversified types of games for young people, the game types for the elderly are more relaxed and easy to play, such as card games, fruit cutting games and so on. The characteristics of these games are that the pave is slow and the logic of the game is closer to the behavior logic.

In the process of using mobile applications, the elderly people are on the one hand for practical purposes. At this time, the function of the app should be able to quickly help the elderly to find corresponding information and solve practical problems. On the other hand, apps are due to meet the social demand of the elderly, and increase their sense of accomplishment and satisfaction that they are keeping up with the times by using mobile devices, which requires the applications can let the elderly complete the operation process smoothly and provide accurate information they need, and create a sense of pleasure during use.

\section{B. Information needs of the elderly using mobile applications}

From the perspective of the information requirements of the old people, the information requirements of mobile application can be divided into three categories: definite information requirements, fuzzy information requirements and comprehensive information requirements [5].

Clear information requirement refers to the elderly users who have clear goals, know what information they want to find and where they can find it, such as using utility classes app, opening the flashlight, using a calculator for computing results, using a dictionary for translation, etc. There is a clear demand to solve the problem, and the process is relatively simple.

The fuzzy information requirement refers to that the elderly users who have information needs but not clear about the demand information, which is obtained through searching information, also called exploratory seeking [6]. In seeking medical treatment in the app, for example, older people themselves have uncomfortable symptoms and need treatment, but they do not know which doctor to seed and how to treat it.

The Comprehensive information requirement refers to that the elderly users have a comprehensive demand for information, and they want to find information in all aspects with a weak purpose and a wide range of search. Information kind application software can meet the needs of comprehensive information, which requires the elderly to have enough patience to search and browse to obtain useful information. 


\section{Problems of the elderly using mobile applications}

- Complex function modules: Existing apps for elderly users have too many complicated functions and require too many manual steps, older users will lose patience on multiple trips to the next page, and will be prone to errors due to too much time consuming, and they will even drop out of action.

- The interaction logic does not conform to the behavior logic of the elderly: In the interface operation, there are some operations that do not conform to the behavior logic of the elderly, which increases the burden of learning for the elderly. For example, shake cell phone to cancel, double - click to enlarge, three finger operation and other real life relatively rare action. The elderly can complete a single task operation under the help of the interface or the guidance of others, but it is not easy to remember when operating alone, and it is easy to cause learning resistance.

- Interface design is not suitable for the elderly: the existing app interface design is not in conformity with the physical perception of the elderly, such as poor icon clarity, small text, too fast or too slow reaction speed and waiting for the process $\mathrm{w}$ no proper guidance or tips during the waiting period, etc. And this part mainly is what the interface designer needs to consider.

\section{INTERACTIVE DESIGN METHOD FOR THE ELDERS MOBILE APPLICATIONS}

The elderly have abundant life experience and have relatively sensitive psychological characteristics. The related product design will be affected by user behavior, demand, purpose, use environment and other aspects. Therefore, for the elderly users, it is not only necessary to understand their functional requirements, but also to pay attention to their cognitive characteristics and conduct interactive design accordingly. Interaction architecture includes information architecture, logical structure, and interaction [7]. From these three aspects, we can get the following three interactive design methods for the elderly.

\section{A. Elderly-Oriented Information Architecture}

Information architecture refers to the combination structure of system organization, navigation and classification tags in the information environment. Information architecture focuses on design organization classification, navigation structure, tagging system, search system, and also affected by content, user and situation [8]. The role of information architecture is to reduce the difficulty of finding information, so that users can browse and obtain relevant content efficiently.

The understanding ability, learning ability and memory ability decline with age. When elderly users are using application, their limited capacity of information processing decides that there will have obstacles such as slow understanding, misunderstanding and inability to think with long hours. At the same time, considering that the information needs of the elderly are mainly clear information requirements, elderly users have limited target objects and need to perform simple tasks, and the user behaviors are relatively monotonous. Therefore, the application of elderly users should have simple function, clear logic, single thread operation, and reduce the width and width of information architecture, which is a simplified interactive model.

Information architecture for the elderly should be as follows:

\section{1) Organization system}

The organizational system should be based on hierarchy, while balance depth and breadth, and information organization and structure design should be done at the single page level. In the application of the comprehensive requirements for information, it should be based on a wide and shallow hierarchical structure, so that it is not confusing for the elderly users to return to the main level at any time. In the case of life class, tool class and so on, it should be based on narrow and shallow hierarchical structure.

\section{2) Navigation system}

The memory of old people is easy to interrupt, therefore the navigation system for older users should to build the scenarios to let older users know which application they are in and which position they are in the system, and finally found the answer to the question. At the same time, the hierarchical structure is used to guide the elderly users to reach the destination with the shortest path and avoid getting lost in the journey to find the answer.

\section{3) Label system}

The construction of a systematic label should refer to the mainstream label to reduce the burden of relearning by the elderly. When using the icon label, try to add text assistance to avoid the old people struggling with the meaning of the icon. In addition, minimize the range of possible meanings of tags to make it easier for older users to identify. In a word, the labelling system should be in accordance with the language habits of the elderly while reflecting its contents.

\section{4) Search system}

First determine if a search system is required, then use keywords to indicate possible search content in the application that requires the search system. The setting of related words for search should be determined, if there are time and budget, by executing user tests, if not, it is best to rely on old people's common sense. This is more conducive to the elderly users, whether in the clear search requirements or fuzzy search requirements, can quickly find the corresponding destination in the application through the keyword of the prompt. 


\section{B. The Interaction Logic Matches the Behavior Logic of the Elderly}

Interaction is made up of "action" and the corresponding "feedback". Action here refers to the conscious behavior, including the person who performs the action, the purpose of the action, the means or tools of the action and the scene of the action. The logic of behavior, as opposed to the physical logic that emphasizes the rational disposition of the property, is based on the rational organization of human behavior [9].

Interaction design is a user-centered design activity to fully consider the user's psychology and behavior. Plenty of experience are accumulated in long-term life of the elderly, they have inherent user experience and behavior. In the process of transferring from interactions with people or product to interactions with smart applications, the elderly retained a large number of life behavior logic, in the short term it is difficult to change again. Therefore, in the interaction design of mobile applications for the elderly, interactive logic should match the elderly behavior logic, including information architecture matching the elderly behavior logic and user interface matching the elderly behavior logic.

Information architecture matching the behavior logic is that when we are in the design of information architecture, we should first understand the motivation of older users and the target of product, then comply with the elderly logical thinking habit and behavior, and finally build information architecture which matches the behavior logic of the elderly.

Interface matching the behavior logic is that the interface conforms to the old behavior logic methods, such as in real books, turning to the left is the next page, turning to the right is back to the previous page, thus the corresponding operations on the interface should be that swiping left to go to the next page and swiping right to back to the previous page.

\section{Multi-Channel Interactive System}

Humans and machines interact through three simple and natural interaction channels of vision, sound and mechanical contact [10]. The interaction design for young people using the visual effect channel can basically complete most of the functions, and the interaction channels of phonetic and mechanical contact are used as an auxiliary and synergistic ways. The elderly universally have phenomenon of vision, hearing and touch recession, therefore, using interaction mechanism of multiple interactive channels can meet the needs of elderly with different cognitive abilities.
The multi-effect interaction channel designed for elderly users should be as follow:

\section{1) Visual interaction}

Mobile application interface designed for elderly users should be simple, easy to understand and easy to recognize. Firstly, the choice of font and font size should be taken into consideration for the reduction of visual and recognition ability of the elderly. Second, the icon design should be concise and clear, containing accurate information to avoid doing ambiguity design which would misleading the elderly to operate. Thirdly, the choice and collocation of color should take into account the comfort level of the aged, using strong color or high contrast colors to attract the attention of the elderly to important information.

\section{2) Voice interaction}

As an important way of natural auditory interaction, voice interaction includes natural speech, music and speech. Voice has information that is easy to understand and is an effective way to transmit orders and provide feedback [11]. For instance, a short and sharp voice warning or melodious music prompting that the action has been completed. These can help the elderly to better understand the needs of the action. In addition, excessive screen time of the elderly can easily cause visual fatigue, and voice interaction can greatly relieve the visual fatigue of the aged, and reduce the error operation caused by visual fatigue.

\section{3) Haptic interaction}

Haptic interaction, on a mobile phone, comprises of physical buttons such as the phone's home button, the volume key and the shutdown button, and the mobile phone's vibration feedback. The machine senses the input information of the user's contact channel, and makes the vibration in time to feed the results back to the elderly users, so as to realize the communication between the elderly and the intelligent mobile terminal. The elderly have years of experience of living, mostly depend on touch system. To add touch interaction in mobile applications in line with the tactile experience of old people in the real world, so that the experience of using applications for the elderly is more real and more intuitive.

\section{CONCLUSION}

Start with the cognitive ability of the elderly, this paper analyzes the characteristics of the cognitive ability of elderly users. The design requirements for the interaction design of mobile phone application are put forward in view of the decline characteristics of the elderly in comprehension and executive ability. Interaction design for the elderly, should be paid more attention to simplify the information architecture, interaction logic conforms to the old behavior logic, as well as the interaction effect more channels, therefore can better improve the elderly's experience of using a mobile application, helping the elderly to experience convenient intelligent life and better integrate into this intelligent modern society. 


\section{REFERENCES}

[1] Inmaculada Plaza, Lourdes Martín, Sergio Martin, Carlos Medrano. Mobile applications in an aging society: Status and trends [J]. The Journal of Systems \& Software, 2011,84(11).

[2] CHEN Yong-min, LUO Yong-dong, "Modern Cognitive Psychology: Human Information Processing [M],” Beijing: Tuanjie Press, 1989. (In Chinese)

[3] LIU Dan-dan, CAO Ju-jiang, "Reconstruction of Elderly Toy Design Tactics Based on Cognitive Aging Characteristics[J]," Packaging Engineering, 2012,33(04):59-63. (In Chinese)

[4] LIN Chong-de. Developmental Psychology (Second Edition). The People's Education Press, 2009. (In Chinese)

[5] WEI Yu-long, The Research on Information Architecture of Mobile Tablet Application Based on Cardsorting [D]. Hunan University, 2013. (In Chinese)

[6] ZHANG Yun-qiu, An Wen-xiu, Feng-jia. "Research on search behavior of exploratory seeking. " Library and Information Service, 2014.14. (In Chinese)

[7] DONG Jian-ming, FU Li-min, RAO Pei-lun, Gavriel Salvendy, "Human-Computer Interaction: User Centered Design and Evaluation (Fourth Edition) [M]." Beijing, Tsinghua University Press, 2013. (In Chinese)

[8] Louis Rosenfeld,Peter Morville,Jorge Arango, Information Architecture: For the Web and Beyond, 4E, Beijing, China Industry and Information Technology Publishing, 2016. (In Chinese)

[9] XIN Xiang-yang, "Interaction Design: From Logic of Things to Logic of Behaviors [J]," Art \& Design, 2015(01):58-62. (In Chinese)

[10] ZHAN Hong-bing, TU Da-Wei, ZHANG Guo-dong, JIANG Ji-Liang, "Man-machine Interaction Effect Channel and Interface Design Suitable for Cognitive Change of Old People [J]," Chinese Journal of Ergonomics, 2013,19 (01): 67-71+92. (In Chinese)

[11] Alan cooper. The Inmates Are Running the Asylum: Why High Tech Products Drive Us Crazy and How to Restore the Sanity. Carmel, Sams Publishing, 2004. 